\title{
Sociedad de la Información, Sociedad Digital, Sociedad de Control
}

\author{
Information Society, Digital Society, Control Society
}

Andoni Polo Roca · andpoloro@gmail.com

Enviado: $17 / 04 / 2020$

Aceptado: 28/05/2020

\section{Resumen}

Las nuevas tecnologías y la actual digitalización han traído un cambio en la información. El aumento en el tamaño, cantidad y velocidad de los datos, así como la aparición del Big Data, han supuesto una revolución digital. La relación constante con la tecnología y la digitalización ha reforzado la Sociedad de la Información, al multiplicar su esencia misma, y, además, ha impulsado la Sociedad Digital: un modelo de sociedad que se caracterizará por desarrollarse, en su mayor parte, en el espacio virtual o digital. Dicho espacio virtual será impulsado por avances tecnológicos como el Internet of Things, los dispositivos inteligentes y otros instrumentos que supondrán un cambio en la estructura de la sociedad (relaciones sociales, laborales, organizativas, educativas, etc.). Sin embargo, la constante interacción con el espacio virtual y las nuevas tecnologías, provocarán que toda nuestra información quede al alcance de cualquiera (la huella digital, información personal, hábitos, etc.), que podrá ser utilizada con diversos fines, lo que supondrá fenómenos como la geolocalización, el tratamiento de macrodatos y demás. De este modo, la consecuencia será la existencia de una vigilancia líquida o vigilancia digital, basada en toda la información al alcance de cualquiera (bautizado ya como dataveillance o vigilancia de datos), que derivará en un panóptico digital y, a su vez, en una Sociedad de Control.

Palabras clave: sociedad digital; espacio virtual; panóptico digital; vigilancia digital; sociedad de control.

\section{Laburpena}

Informazioa aldatu egin dute teknologia berriek zein egungo digitalizazioak. Datuen tamaina, kantitate eta abiaduraren hazkundeak, bai eta Big Dataren agerpenak, iraultza digital hutsa ekarri dute. Teknologia eta digitalizazioarekin dugun etengabeko harremanak indartu egin du Infomazio-Gizartea, bere oinarri bera ugaldu egin delako, eta, era berean, Gizarte Digitala bultzatu du: espazio birtual edo digitalean gauzatuko den gizarte eredua. Internet of Things, gailu adimendun eta beste horrenbeste tresnek bultzada emango diote espazio birtualari, gizartearen egitura bera aldatuko dutenak (harreman sozialak, lan-harremanak, antolakuntza, hezkuntza, etab.). Espazio birtualarekin eta teknologia berriekin dugun etengabeko interakzioak, haatik, gure informazioa edonoren esku jarriko dute (aztarna digitala, informazio pertsonala, ohiturak, etab.), xede anitzekin erabil daitekeen informazioa, eta, hala, geolokalizazio, makrodatuen tratamendu eta horrenbeste fenomeno agertuko zaizkigu. Horren ondorioa zaintza likido edo zaintza digital baten existentzia izango da, edonoren esku dagoen informazioan oinarri izango duena (dataveillance edo datu-zaintza izendatu dena), eta horrek panoptiko digitala ekarriko du eta, horri loturik, Kontrol-Gizarte bat.

Gako-hitzak: gizarte digitala; espazio birtuala; panoptiko digitala; zaintza digitala; kontrol-gizartea. 


\begin{abstract}
New technologies and current digitization have meant a change in information. The increase in the size, amount, speed and range of data, as well as the appearance of Big Data, have brought about a digital revolution. The constant relationship with technology and digitization has reinforced the Information Society, multiplying its very essence, and, in addition, has promoted the Digital Society: a model of society characterized by developing, for the most part, in virtual or digital space. This virtual space will be driven by technological advances such as the Internet of Things, smart devices and other instruments that will mean a change in the structure of society (social, labor, organizational, educational relations, etc.). However, the constant interaction with virtual space and new technologies will cause all our information to be available to anyone (fingerprint, personal information, habits, etc.), which can be used for various purposes, which will mean phenomena such as geolocation, big data processing and others. This way, the consequence will be the existence of a liquid surveillance or digital surveillance, based on all the information available to anyone (what has been called dataveillance), which will lead to a digital panopticon and, in turn, a Control Society.
\end{abstract}

Keywords: digital society; digital panopticon; digital surveillance; virtual space; control society.

\title{
1. INTRODUCCIÓN
}

Vivimos en la era de la información. Es innegable. La información es la base de la sociedad actual y esta ha penetrado en todos y cada uno de los aspectos de la sociedad. La información, en general, y, sobre todo, la información estratégica, tan vieja como el espionaje, es fuente del nuevo poder (Keohane y Nye, 1998). Tanto la comunicación como la información en las sociedades actuales suponen el comienzo de una nueva existencia y de una nueva era: la de la información (Castells, 1997: 514).

Los avances en las tecnologías de información y comunicación (TIC) son la base principal de la denominada Tercera Revolución Industrial, que supuestamente traería consigo un cambio radical en los patrones y sistemas del conocimiento, en los modos de producción, esquemas económicos, relaciones sociales y culturales (Lafuente y Genatios, 2006: 21); así, las TIC y las tecnologías digitales han supuesto un impulso gigante para la Sociedad de la Información.

Con las nuevas tecnologías, además, la información se multiplicará; tendremos un cambio sustancial en la escala, el tamaño y el tipo de datos. Ello nos lleva a la aparición de fenómenos como el Big Data y demás dispositivos inteligentes que recogen y almacenan millones de datos personales de cada integrante de la sociedad; ello implica que la información de cada persona esté a disposición de cualquiera en la red. Ya se calculó que para el 2018 habría más de 3.330 millones de dispositivos conectados en las ciudades inteligentes (CCOO, 2017), compartiendo millones y millones de datos entre ellos cada segundo. Actualmente, las empresas ya citan a los datos como uno de los recursos clave y un factor distintivo fundamental para los negocios; los datos han dejado de ser un recurso escaso para convertirse en un recurso fundamental, renovable y cada vez más abundante (BSA, 2015). Existen ya hasta mercados de información personal (Contreras Gómez, 2017: 162).

En este contexto, los integrantes de la sociedad actuaremos como productores de la misma y ello puede llevarnos a distintos problemas; la cantidad de datos que proporcionamos en 
la red y dejamos accesibles al público nos pueden llevar a situaciones de control social, en el que el Big Data acaba siendo un gran hermano (Pineda de Alcázar, 2018). Uno de los ejemplos puede ser el de la geolocalización o localización geográfica de los integrantes de la sociedad mediante sus dispositivos digitales por cuenta de empresas privadas ${ }^{1}$ o, incluso, de los poderes públicos, como la situación que trajo la COVID-19 en el año 2020².

De este modo, en esta era marcada por la transformación que han supuesto tanto la información, como los avances tecnológicos y digitales, la sociedad se caracteriza por ser una Sociedad de la Información; pero ello puede llevarnos a la aparición del control social y una evolución del panóptico y la vigilancia a partir de la información, y el cambio en el tamaño y cantidad de datos e información.

Este trabajo, de carácter teórico, responde a la necesidad de analizar las consecuencias sociales del impulso en la información (los datos) por parte de las nuevas tecnologías y su incidencia en la aparición de nuevos modelos de sociedad; todo ello con base en el gran volumen de datos e información que serán el pilar de este trabajo. La hipótesis radicará en cómo la información que caracterizó la Sociedad de la Información está suponiendo actualmente, a consecuencia de la digitalización, la caracterización de una nueva sociedad donde el mismo protagonista (la información) será el pilar de la misma.

La estructura del estudio se dividirá en tres partes. La primera, el esbozo de la Sociedad de la Información, la que está hoy más presente que nunca, ya que se ha reforzado la información (calidad, cantidad, etc.); la segunda, las consecuencias sociales de la digitalización y nuevas tecnologías que moldearán la Sociedad Digital, y, por último, el efecto que todo ello está teniendo en la sociedad actual: serán la información y los macrodatos los que incrementarán el nuevo panóptico y la nueva vigilancia, y ello derivará en una Sociedad de Control emergente. Así, el marco teórico de la investigación, que seguirá una metodología eminentemente teórica, se centrará en la información en tres fases: mera información, información en la digitalización e información como control social.

\section{SOCIEDAD DE LA INFORMACIÓN Y SOCIEDAD DEL CONOCIMIENTO}

Como hemos mencionado, actualmente estamos ante la Sociedad de la Información; por ello, nos es necesario poner el acento sobre las características de este modelo de sociedad,

\footnotetext{
1 Véanse: Toledano, B. (2018). La app de La Liga usa tu móvil para saber qué bares emiten fútbol sin licencia. El Mundo, 2018. Accesible en: https://www.elmundo.es/tecnologia/2018/06/11/5b1e3b4e22601de31d8b4613. html; Sánchez, J. L. (2018). La Liga de Fútbol usa el micrófono del teléfono de millones de aficionados para espiar a los bares. El Diario.es, 2018. Accesible en: https://www.eldiario.es/tecnologia/Liga-Futbol-microfonotelefono-aficionados_0_780772124.html y Raya, A. (2020). Google sabe si estamos saliendo esta cuarentena: lanza un informe de movilidad del COVID-19. El Español, 2020. Accesible en: https://www.elespanol.com/ omicrono/software/20200403/google-saliendo-cuarentena-lanza-informe-movilidad-covid-19/479702576_0.html 2 Luis Sanz, J. (2020). Europa usará los datos de localización de nuestros móviles para luchar contra el Covid-19. El País, Cinco Días. Accesible en: https://cincodias.elpais.com/cincodias/2020/03/26/ lifestyle/1585221347_969252.html
} 
ya que es nuestro punto de partida. Asimismo, si bien es cierto que la Sociedad de la Información y la Sociedad del Conocimiento se han tomado como sinónimos, nos será preciso hacer algunas matizaciones que nos pueden llevar a distinguir ambas.

\subsection{La Sociedad de la Información ${ }^{3}$}

La Sociedad de la Información hace referencia a la importancia social que se le concede a la comunicación y la información en la sociedad actual, donde se involucran las relaciones sociales, económicas y culturales (Alfonso Sánchez, 2016: 236); con esta fórmula se quiere aludir al papel absolutamente central que la información y la comunicación revisten en el interior de la organización social, del mundo productivo, de los procesos políticos y de todo mecanismo decisorio (Toniatti, 1991: 2 y 3). Ante una sociedad preindustrial, analfabeta y jerárquica, de estructura ocupacional rígida y escasa densidad de comunicaciones, se desarrolla una sociedad cada vez más industrial, básicamente alfabetizada, no jerárquica, de participación ciudadana e intensamente comunicada (Blázquez Entonado, 2001: 25). Esta consideración permite intuir que no es posible concebir la conformación de una sociedad sin la influencia de la información (Alfonso Sánchez, 2016: 236).

En la Sociedad de la Información resulta elemental el proceso de captar, procesar y comunicar las informaciones necesarias (Cervantes Benavides, 2007: 27 y 28). Así, en este tipo de sociedad, la gran mayoría de ésta se dedicará a la prestación de servicios y dichos servicios consistirán en el procesamiento, distribución o utilización de la información (Castells, 1997: 43); los servicios estarán relacionados con la información y esta será el eje de la sociedad. La ciencia y la tecnología, asimismo, se convertirán en fuerzas inmediatas de producción (Aristovnik 2014: 55).

\footnotetext{
3 Sociológicamente, Castells prefiere el término «Sociedad Informacional» antes que «Sociedad de la Información»: aunque el conocimiento y la información sean elementos decisivos, «Sociedad de la Información» «destaca el papel de esta última en la sociedad en cambio» y, en cambio, "el término informacional indica el atributo de una forma específica de organización social en la que la generación, el procesamiento y la transmisión de información se convierten en las fuentes fundamentales de la productividad y el poder, debido a las nuevas condiciones tecnológicas que surgen en este período histórico» (Castells, 1997: 47); por ello, el sociólogo defiende que es preferible el término «Sociedad de la Información», antes que «Sociedad de la Información» (pone como ejemplo la distinción entre industria e industrial).

Desde la óptica de la lingüística, asimismo, resulta preferible también «Sociedad Informacional» antes que «Sociedad de la Información». El primer término («Sociedad de la Información») constituye un sintagma nominal donde «Sociedad» es el núcleo nominal y «de la Información» un sintagma preposicional (SP) o construcción preposicional (CP) con un nexo subordinante: la preposición «de». El segundo término («Sociedad Informacional»), en cambio, constituye también un sintagma nominal, pero, en este caso, «Sociedad» es el núcleo nominal e "Informacional» es el modificador o adyacente: un adjetivo calificativo que sirve de complemento de un sustantivo y matiza el núcleo nominal. El adjetivo siempre da más peso al nombre (al núcleo nominal, en el sintagma) que otro complemento; y, aunque es cierto que «Información» es una palabra larga y se vería más clara en su sustantivo que como adjetivo, resulta necesario hacer uso del adyacente o modificador, dado que el adjetivo calificador le confiere más relevancia al nombre en el sintagma y es eso lo que queremos conseguir en el presente caso: darle protagonismo absoluto a "Sociedad», ya que resulta el núcleo del análisis sociológico, y conseguir que la información sea únicamente un término que describa o califique dicho objeto del estudio, es decir, que describa el tipo de sociedad actualmente imperante, la «Sociedad Informacional». En suma, parece que sería más correcto utilizar el término "Sociedad Informacional», ya que la sociedad no pertenece a la información, sino que la información es un término que describe o califica la sociedad, haciendo referencia a una sociedad en la cual la información es el eje principal. Podemos fijarnos, por ejemplo, en su versión en inglés: "Information Society», y no «Society of Information»; o en euskera: "Informazio-Gizartea», y no «Informazioaren Gizartea».
} 
En lo que respecta a los orígenes o primeros pasos del concepto, en 1914 surgió el concepto de sociedad post-industrial y Arthur J. Penty defendió que la evolución de la sociedad pasaría por el intercambio de información entre personas, mediante la creación de asociaciones, gremios y otros (Penty, 2004).

Uno de los primeros autores en desarrollar el concepto de Sociedad de la Información fue el economista Fritz Machlup (1962), a principios del siglo XX (Crawford, 1983: 380 y 381), a quien se le atribuye el uso del término Sociedad de la Información por primera vez (García Manjón, 2010: 19), con el trabajo La producción y distribución del trabajo en los Estados Unidos, en el que estudió la tipología de trabajos en EEUU y observó cómo los empleos estaban pasando a estar relacionados con la información. En dicho trabajo, distinguió cinco sectores del conocimiento: educación, investigación y desarrollo (I+D), medios de comunicación, tecnologías de la información y servicios de la información; dando paso, de este modo, a la industria del conocimiento (Machlup, 1962; Rooney 2014: 261). El trabajo fue ampliamente reconocido y traducido a varios idiomas, entre ellos el japonés, y, así, sociólogos japoneses como Kamishima (Kuwahara et al., 1964) ya se referían también a la Sociedad de la Información o Jōhōka shakai.

El sociólogo estadounidense Daniel Bell, por otro lado, siguió la tesis de Penty. Después de los tres tipos de sociedad que se han sucedido a lo largo de la historia, Bell (1976) distinguió, partiendo de la tesis de Penty, un nuevo tipo de sociedad que sucedía a la sociedad industrial: la Sociedad Postindustrial. Con este tipo de sociedad, Bell defendía que esta se caracteriza por que su eje principal será el conocimiento teórico y los servicios basados en el conocimientos habrán de convertirse en la estructura central de la nueva economía y de una sociedad apuntalada en la información donde las ideologías resultarán sobrando; encontrándonos, por lo tanto, ante una sociedad que se basa en la economía de la información, en contraste a la economía de las mercancías, sin que una sustituya a la otra, coexistiendo ambas. Así, según el sociólogo americano, una sociedad post-industrial es básicamente una sociedad de la información; el intercambio de información en términos de varios tipos de procesamiento y almacenamiento de datos, investigación de mercado, etc. es la base de la mayoría de cambios económicos (Bell, 1976).

Por otro lado, Masuda (1981 y 1984) estableció las características de este tipo de sociedad, en las que las condiciones de producción se deben, fundamentalmente, a la generación y transmisión de información como principal producto de las economías avanzadas y, sobre todo, a la transición de los medios de generación de riqueza de los sectores industriales a los sectores de servicios en los que la información se convierte en el activo más importante (Casas Pérez, 2010).

En cuanto a la relación de la Sociedad de la Información con la tecnología, debemos apuntar a que ambos conceptos van unidos, pero la tecnología no es el pilar de este tipo de sociedad (lo es la información), ya que, tal como criticó Webster (1994), en sus cinco definiciones de la Sociedad de la Información - tecnológica, económica, ocupacional, espacial y 
cultural—, si el elemento principal de este tipo de sociedad es la tecnología, el concepto debería llevar la palabra tecnología en su denominación, pero no es así. De igual modo, tal como afirma Castells (2007), al afirmar que la era de la información (también denominada era informática) supone el periodo histórico caracterizado por una revolución tecnológica centrada en las tecnologías digitales de información y comunicación, concomitante, pero no causante; así, la tecnología aparecerá conjuntamente con la Sociedad de la Información, pero no será su causante.

De este modo distinguimos que la sociedad cuyo eje es la información (Sociedad de la Información) tuvo un primer momento, a principios del siglo XX, que fue impulsado por los mass media (televisión, radio y prensa) y, un segundo, con la aparición de las TIC que supusieron la tecnologización y la integración multimedia de viejas tecnologías de la información (radio, teléfono, cine, prensa y TV) a partir del desarrollo de la informática (Salvat Martinrey y Serrano Martin, 2011).

Con el auge de las nuevas tecnologías y la revolución digital, la Sociedad de la Información está hoy más viva que nunca: el incremento en el volumen, velocidad y el tipo de información han reforzado y revitalizado la información, que es la esencia misma de este modelo de sociedad. Como hemos dicho anteriormente, la distribución y procesamiento de la información caracteriza a este tipo de sociedad, y con la tecnologización y digitalización actual, la Sociedad de la Información se ha consolidado, desarrollado y revitalizado: la información es el petróleo del siglo xxI.

\subsection{La Sociedad del Conocimiento}

Por su parte, el término Sociedad del Conocimiento (Knowledge Society) fue introducido por Drucker (1969 y 1993) en el año 1969 y fue abriéndose paso durante los años 70, junto a la Sociedad de la Información y las nuevas tecnologías, consolidándose en el siglo xx como un nuevo paradigma. Siguiendo el planteamiento de Sanz-Magallón, podemos definir la Sociedad del Conocimiento como «aquélla en que los ciudadanos disponen de un acceso prácticamente ilimitado e inmediato a la información, y en la que ésta, su procesamiento y transmisión actúan como factores decisivos en toda la actividad de los individuos, desde sus relaciones económicas hasta el ocio y la vida pública» (Sanz-Magallón, 2000).

Cuando Bell (1976) caracterizó la Sociedad Postindustrial, aludió al conocimiento como el axial principal de dicha sociedad y defendió que la información y el conocimiento son transversales y los encontramos detrás de todos los aspectos que definen la sociedad postindustrial; el conocimiento científico y tecnológico serán la espina vertebral de estas sociedades. Bell (1976: 175) definió el conocimiento como una serie de afirmaciones organizadas de hechos o ideas que presentan un juicio razonado o un resultado experimental, que se transmite a los demás mediante algún medio de comunicación en alguna forma sistemática. Según Porat (1977: 2), la información son los datos que se han organizado y comunicado. 
En este sentido, debemos distinguir conocimiento e información: la información es algo externo que se encuentra a nuestra disposición; el conocimiento es un crecimiento interno, un avance hacia nosotros mismos, una potenciación de nuestra capacidad operativa (Marrero Rodríguez y Santiago García, 1999). Confundir información con conocimiento es el error primordial de confundir el modo de ser de las personas con el modo de ser de las cosas (Llano, 2000). Así, debemos diferenciar este tipo de sociedad de la Sociedad de la Información, diferenciando, de igual modo, el conocimiento y la información. La información será instrumento del conocimiento, ya que la información engloba datos, y cuando los integrantes de dicha sociedad acceden a estos y los conocen, nos encontraremos ante el conocimiento y este tipo de sociedad. Según Castelfranchi (2007), la Sociedad del Conocimiento se distingue de la Sociedad de la Información en que la primera sirve para transformar la información en recursos que permiten a la sociedad tomar medidas efectivas, mientras que la segunda solo crea y difunde los datos en bruto.

El teórico en filosofía de la comunicación y medios de difusión y subdirector general de la UNESCO para la Comunicación y la Información, Abdul Waheed Khan, expone así la diferencia entre ambas caracterizaciones de la sociedad (UNESCO, 2003):

Los dos conceptos son complementarios. La Sociedad de la Información es la piedra angular de las Sociedades del Conocimiento. Mientras que el concepto de «Sociedad de la Información» está vinculado a la idea de «innovación tecnológica», el concepto de «Sociedades del Conocimiento» incluye una dimensión de transformación social, cultural, económica, política e institucional, y una perspectiva más pluralista y de desarrollo. [...] el conocimiento es importante [...] para el empoderamiento y el desarrollo de todos los sectores de la sociedad. Por lo tanto, el papel de las TIC se extiende al desarrollo humano en general, y al aprendizaje permanente.

La generación de conocimiento implica la existencia de información previa, pero requiere de la experiencia y el aprendizaje previos en términos de asignación de sentido, que son los que le generan su verdadero valor; sin embargo, en términos de asignación el valor social, la transmisión de información no es garantía de generación de conocimiento (Casas Pérez, 2010). Por lo tanto, tratar como iguales a la información y el conocimiento deja en la sobra a actividades y capacidades humanas muy fundamentales, como el aprendizaje y la cognición. (Steinmueller, 2002). Para que exista conocimiento, es necesario, no únicamente recibir la información para conocerla, sino también que el sujeto traduzca la información y la interprete como verdadera aportándole cierta carga de significación social, la diseminación de la información no garantiza por sí sola el conocimiento (Casas Pérez, 2010).

En la Sociedad del Conocimiento, además, se debe garantizar el derecho de toda la sociedad a tener acceso a la información a fin de poder comunicarse y producir conocimiento (Hamelink, 2003); pero tener acceso a la información o poseerla no necesariamente implica que la persona aprovechará las oportunidades en beneficio propio para mejorar su 
nivel de vida, esto implica precisamente el desarrollo de competencias (Sen, 2000). En este tipo de sociedad, la ciudadanía accede a dicha información y la conoce, teniendo la información efecto sobre cada aspecto de vida de los integrantes de la sociedad (Sanz-Magallón, 2000). De este modo, puede darse el caso de estar ante una Sociedad de la Información, pero no ante una Sociedad del Conocimiento: bien porque parte de la sociedad no tiene acceso a dicha información o bien porque, aun teniendo acceso, no la traduce e interpreta de forma que influya en su vida diaria y genere conocimiento.

En cuanto a la aparición de este tipo de sociedad, según Sanz-Magallón (2000), la sociedad del conocimiento surge como consecuencia de los cambios que inducen en la sociedad una serie de innovaciones tecnológicas desarrolladas en tres sectores convergentes: la informática, las telecomunicaciones - y en especial Internet-y los medios de comunicación; Castells (2002a) incluye también la ingeniería genética. De este modo, la información que contendrá los datos en bruto y los integrantes de dicha sociedad accederán a esa información, a esos datos, y los conocerán; todo ello teniendo como herramienta todos los medios tecnológicos, como las TIC. Dichas herramientas tecnológicas, según Echevarría, son un medio para distribuir información, pero, sobre todo, un medio de conocimiento y de saber capaz de modificar nuestra memoria, nuestros comportamientos y nuestra identidad personal y social (Echevarría, 1999; 2000).

\section{LA SOCIEDAD DIGITAL O VIRTUAL}

\subsection{La transformación informática, tecnológica y digital: 1980-2010 y 2010 en adelante}

En primer lugar, debemos hacer una panorámica histórica sobre la informática y revolución tecnológica, para analizar las implicaciones sociales que tuvo y poder distinguir los diferentes modelos sociales.

Desde la óptica histórica, nuestro primer acontecimiento reseñable son los primeros ordenadores, creados en torno a 1950 (denominados «ordenadores de primera generación»); le sucedieron los ordenadores de segunda, tercera, cuarta y quinta generación $\mathrm{n}^{4}$. Sin embargo, el comienzo de la etapa de expansión será alrededor del año 1980, con la llegada y comercialización de los ordenadores domésticos 5 . En la evolución tecnológica, de este modo, en

\footnotetext{
${ }^{4}$ Al hablar de ordenadores de primera generación nos referimos a ordenadores electromecánicos; el primer ordenador podemos localizarlo en torno a 1941, que llegó de mano del alemán Konrad Zuse. El comienzo de los ordenadores de segunda generación la encontramos en 1958 y de los ordenadores de tercera generación a mediados de los años 60. Después llegaron los ordenadores de cuarta generación en torno a 1971 y los de quinta generación en 1990.

${ }^{5}$ En 1975, la invención del chip redujo los ordenadores a los tamaños actuales, haciendo posible la comercialización de los ordenadores domésticos; en España, por ejemplo, ya era posible comprar ordenadores domésticos desde 1979. En Europa, la empresa Sinclair Research presento el primer ordenador de la historia que constaba menos de 100 libras; de este modo, los ordenadores comenzaron a ser accesibles para las familias trabajadoras. Los primeros años de la informática doméstica en España sería entre 1980 y 1985.
} 
lo que respecta a los ordenadores y sus consecuencias desde el punto de vista sociológico, distinguimos un primer momento (1950-1980) y un segundo momento (1980 en adelante), siendo ese segundo cuando los ordenadores comenzaron, poco a poco, a tener su espacio en la sociedad.

Por el otro lado, tenemos Internet. Internet es una herramienta social que contiene miles de millones de datos al alcance de cualquiera. Internet no es una energía más; es realmente el equivalente a lo que fue primeramente la máquina de vapor y luego el motor eléctrico en el conjunto de la revolución industrial (Castells, 2002a).

Internet, tal como lo conocemos hoy, apareció en los años 90. Sin embargo, sus inicios se remontan a los años $60^{6}$, financiado por el Departamento de Defensa de los Estados Unidos, como un programa militar sin aplicación militar (Castells, 2002b). El inicio del actual Internet en los años 90 se dio por tres principales factores (Castells, 2014): el descubrimiento de la tecnología de la red de redes (World Wide Web), el cambio institucional en la gestión de internet (se privatiza y pasa a estar en manos de la comunidad global de internautas) y los cambios significativos en la estructura, la cultura y la conducta social (la comunicación en red como forma predominante de organización). Englobamos, así, el momento de la revolución tecnológica del año 1980 en adelante: cuando los ordenadores empezaron a ganar peso e Internet se expandió. De este modo, en la década de los 2000 tendrá lugar la expansión mundial de los ordenadores y de Internet; por tanto, englobamos el periodo 1980 a la década de los 2000.

Después del auge de Internet (1990-2010), la década de 2010 será la del uso generalizado y la interconexión de dispositivos de las redes móviles y la telefonía móvil, recursos y sitios de Internet; y también la de los smart devices o dispositivos inteligentes, el Internet of Things, servicios online, etc. Para mitad de la década (2015), habrá una gran interconexión entre cualquier tipo de aparato inteligente (móviles, MP4, televisores, ordenadores, etc.), una gran interconexión entre dispositivos que crearán una red ubicua; además de superar una simple interconexión entre terminales y estar ante la «nube» (cloud computing, computación en la nube, servicios en la nube o informática en la nube).

En esta etapa, a diferencia de la anterior (1990-2010), la sociedad se transformará digitalmente, avanzando hacia los servicios habituales en la red (compraventa, música, cine, servicios sanitarios, etc.) y las relaciones entre las personas pasarán a formato digital con el auge de estas conexiones, nube y ciberespacio. Las relaciones laborales también cambian: la digitalización de muchos puestos de trabajo o la posibilidad de teletrabajo se expandirá en la sociedad. En este nuevo contexto - como consecuencia de los dispositivos inteligentes interconectados, actividades cotidianas realizadas de forma digital, ciberespacio, etc.-, la sociedad comenzará a vivir de forma digital.

\footnotetext{
${ }^{6}$ En 1969 se creó una pequeña red llamada ARPANET con cuatro ordenadores. Ese proyecto fue creciendo y
} aparecieron otros proyectos como MILNET (para usos militares), NSFNET (usos civiles), etc. 
INGURUAK [68] | 2020 | 35-77

Sociedad de la Información, Sociedad Digital, Sociedad de Control | Andoni Polo Roca

\subsection{De la Sociedad Red a la Sociedad Digital}

En toda esta revolución informática, y partiendo de la Sociedad de la Información, Castells (1997) caracterizó lo que denominó la Sociedad Red (network society) como la traducción social que tuvo la primera; es decir, lo que supuso sociológicamente la Sociedad de la Información. Es así como Castells la define (IIS, 2001):

La sociedad red es la estructura social característica de lo que se ha estado llamando durante años «Sociedad de la Información» o "Sociedad postindustrial». Tanto «Sociedad postindustrial» como «Sociedad de la Información» son términos descriptivos que no proporcionan la sustancia, no son lo suficientemente analíticos. [...] La sociedad red es una sociedad donde las estructuras y actividades sociales clave se organizan en torno a redes de información procesadas electrónicamente. [...] no se trata solo de redes o redes sociales, porque las redes sociales han sido formas muy antiguas de organización social. Se trata de redes sociales que procesan y gestionan información y están utilizando tecnologías basadas en microelectrónica.

Este nuevo tipo de sociedad, según el sociólogo, se fue formando en las dos últimas décadas del siglo xx (Castells, 2020) y ha ido transformando toda la actividad humana, siendo una de las mayores revoluciones de la historia, especialmente desde el nuevo milenio. Las redes digitales van ya más allá de Internet y a ello le sumamos la aparición de la Inteligencia Artificial (IA) con máquinas capaces de aprender y decidir; cada uno de nuestros ámbitos de vida está siendo transformada (Castells, 2020).

De este modo, la Sociedad Red es la forma de organización social sustentada en la información (Castells, 2006); se diferencia, así, la sociedad basada en la información, de la estructura social que esta crea: una sociedad basada en redes de comunicación digitalizada en todos los aspectos de la vida, la política, la economía y las relaciones personales. Igualmente, Van Dijk (2006: 20) define la sociedad de redes como una formación social con una infraestructura de redes sociales y de medios que permite su modo principal de organización en todos los niveles. Cada vez más, estas redes conectan todas las unidades o partes de esta formación (individuos, grupos y organizaciones). Hablamos de un nuevo término (unido a la Sociedad de la Información): un tipo de sociedad donde prolifera y crece Internet que determina nuevos modos de organización, comunicación y de relación social o de ocio (Vázquez Vela, 2011).

Muchas veces se han tomado por sinónimos, pero debemos tener en cuenta que la caracterización de la Sociedad Post-industrial o Sociedad de la Información fue emergiendo desde el principio del siglo xx y, en cambio, la Sociedad Red que Castells caracterizó, se fue creando en las dos últimas décadas del siglo xx. Sin embargo, no podemos tomar a la Sociedad Red como una mera traducción o caracterización social de la Sociedad de la Información, ya que el hecho de que la información fuese el pilar de una nueva sociedad emergente a principios del siglo xx también trajo implicaciones sociales, implicaciones que se 
dieron antes de las últimas dos décadas del siglo xx. Por lo tanto, no podemos afirmar categóricamente que la Sociedad Red sea el aspecto social de la Sociedad de la Información.

Será Internet la base material y tecnológica de la Sociedad Red —al fin y al cabo, Internet es una contracción de «interconnected network» ("red interconectada»)—, su infraestructura tecnológica y medio organizativo (Castells, 2001). Así, las tecnologías de red son el medio de esta nueva estructura social y de esta nueva cultura (Papacharissi, 2010). Por lo tanto, las tecnologías no serán la Sociedad Red, sino que las tecnologías serán su infraestructura. Está será otra distinción entre la Sociedad de la Información y la Sociedad Red, ya que la existencia de la tecnología en la Sociedad de la Información no tiene por qué implicar la existencia de una Sociedad Red, pero, sin embargo, la existencia de esta última sí implicará siempre la existencia de la primera, ya que sin la tecnología no existiría la Sociedad Red (es su infraestructura).

Las relaciones, asimismo, han sufrido una profunda transformación; la irrupción de redes sociales ha supuesto una nueva revolución sociotecnológica en Internet (Castells, 2014), y en estas ya están representadas todas las actividades humanas, que incluyen relaciones personales, negocios, trabajo, cultura, comunicación, movimientos sociales y política (Boyd y Ellison, 2007). Este tipo de sociedad organiza sus relaciones en redes digitales que sustituirán paulatinamente las comunicaciones cara a cara entre personas (Van Dijk, 2006); e, igualmente, en este tipo de sociedad, el poder está contenido en las redes, más que en la calle, las estructuras sociales y actividades principales se organizan mediante redes digitales (Castells, y Cardoso, 1996). Según Barney (2003), de igual modo, este tipo de sociedad tiene dos características básicas: la primera es la presencia de tecnologías sofisticadas, casi exclusivamente digitales, de comunicación en red y gestión o distribución de información y, la segunda, la reproducción e institucionalización en (y entre) esas sociedades de redes como la forma básica de organización y relación humana en una amplia gama de actividades sociales, configuraciones y asociaciones políticas y económicas.

\subsection{La revolución digital, la Sociedad Digital y el espacio digital o virtual}

Tal como exponen Uriarte y Acevedo (2018: 37), si bien la Sociedad Red constituye un marco apropiado para la transformación digital, esto no significa más que un punto de partida. Los cambios derivados de la transformación digital son una cuestión más espinosa que la implantación de nuevas tecnologías o instrumentos; esta transformación digital tiene efectos sobre la conciencia y organización social, y ya podemos hablar de servicios digitales, economía digital o empresas digitales (Uriarte, Acevedo, 2018). Así, las TIC, además de ser el soporte físico de la información, conforman, debido a Internet, una poderosa red y, en consecuencia, se ha comenzado a reconfigurar y crear un entorno muy diferente al mundo físico (Brito Alvarado y Capito Álvarez, 2016: 26).

Es decir, actualmente podemos hablar de una Sociedad Digital (Alcántara, 2008) o Sociedad virtual, Cibersociedad, etc. Este tipo de sociedad supone una evolución en nuestro modo de 
relacionarnos, organizarnos y estructurarnos: nos encontraremos en un espacio virtual, el ciberespacio, donde podremos realizar nuestro día a día, alejándonos del mundo real. Lo digital, de este modo, transformará la sociedad por completo: relaciones laborales, sentimentales y afectivas; poder; comunicación, etc.

Castells (2014), sin embargo, niega alguna diferencia entre una Sociedad virtual y la Sociedad real o física. Según él, la virtualidad en la que vivimos es una de las dimensiones de la realidad: vivimos «con» Internet, no vivimos «en» Internet. No salimos de Internet; Internet está presente en nuestro día a día. La conexión entre lo virtual y lo presencial (o lo real, partiendo de la base que la realidad es virtual y presencial) la hacemos nosotros. Conectamos el «espacio de los flujos» (la virtualidad real) y el espacio de los lugares o espacio real (Castells, 2014).

Sin embargo, en la fase actual de digitalización (2010 en adelante), sí que realizamos nuestro día a día "en» Internet: no es que estemos conectados a la red, es que vivimos en la red. Hoy en día realizamos nuestro día a día digitalmente y la digitalización de la sociedad está suponiendo una sociedad virtual donde realizaremos nuestras actividades cotidianas (laborales, sentimentales, políticas, etc.); ya no estamos interconectados como en una tela de araña, sino que hemos creado un espacio virtual propio, donde, no solo las personas, sino también todos los dispositivos y objetos inteligentes están conectados, algunos incluso con capacidad de pensar y decidir, como la IA. Todo lo cual trae, igualmente, cambios en el modelo de sociedad.

Así, en la sociedad que vivimos actualmente, nos es necesario tener en cuenta el panorama digital o entorno digital, ya que, más allá de las TIC, lo digital ha supuesto un cambio: el nuevo sistema que ha constituido Internet ha creado una instancia nueva, un universo virtual, el ciberespacio (Salvat Martinrey y Serrano Martin, 2011). Así, Salvat Martinrey y Serrano Martin (2011: 46) afirman lo siguiente:

«[...] vivimos en una Sociedad de la Información condicionada por las nuevas tecnologías donde se da la tendencia a prescindir de lo natural, es decir, a la emergencia de un medio artificial nuevo, que llamamos virtual [...] las nuevas tecnologías no se limitan ya a dominar sobre lo natural, es decir, a reproducirlo generando una realidad que siendo natural aparece mediatizada en su reproducción por la máquina, sino que ahora lo que hacen es producirlo directamente generando una realidad nueva.»

De este modo, toda forma de representación virtual es una nueva realidad que se mezcla e interactúa con la realidad del mundo físico (Martire, 2013). Y, así, tal como señalan Harrison y Dourish (1996), los entornos virtuales que encontramos en una pantalla de ordenador producen efectos también en el mundo físico y, por supuesto, no relacionan usuarios virtuales, sino reales. 
Actualmente superamos el marco definido por Webster: en la revolución tecnológica inicial (1990-2010), las relaciones estaban sustentadas por las TIC y, en especial, por la conexión de todos los ordenadores entre sí, lo que posibilita la conexión de terminales desde y entre oficinas, bancos, hogares, tiendas, fábricas, escuelas y todo el globo en sí (Webster, 1994); en dicha interconexión, la red de información, cuyo elemento principal es Internet, podría compararse la red eléctrica o ferrocarril que conectaba a las personas entre sí y facilitaba que la sociedad pasase de miembros alejados o dispersos a miembros unidos o conectados. Hoy en día, en cambio, ya no habrá terminales conectados entre sí, sino que los terminales suponen el acceso al ciberespacio, a la «nube».

Huelga decir que la Sociedad de la Información y la Sociedad Digital coexistirán actualmente, seguiremos estando ante una sociedad con la información como eje principal, aunque no podemos igualar ambos conceptos, ya que la sociedad de principios del siglo xx también era Sociedad de la Información, pero, sin embargo, no era una Sociedad Digital. En esta Sociedad Digital, tendremos no solo información, sino que los servicios básicos serán prestados de forma digital o virtual y nuestras relaciones con otras personas también serán digitales o virtuales.

La Sociedad Digital se caracteriza por: las nuevas tecnologías inteligentes interconectadas que forman una red ubicua (soporte tecnológico del espacio virtual); la creciente capacidad de producir y almacenar información y datos de gran tamaño, y la creación de un nuevo espacio (el espacio virtual), que se diferencia del físico o real, en el que tendrán lugar los distintos aspectos de la vida cotidiana (laboral, económica, social, política, etc.). Estaremos ante una Sociedad virtual o Sociedad 2.0, donde tendremos socialización virtual y relaciones virtuales (on-line, a través de redes sociales, etc.), entretenimiento virtual (servicios en streaming), educación virtual (a distancia, bajo demanda, on-line, etc.), dinero virtual (bancos on-line, criptomonedas, sistema de pago virtuales, etc.), delitos virtuales (ciberdelitos, robos virtuales, etc.) y demás; todo evolucionará al 2.0, desarrollándose en el espacio virtual.

De este modo, las diferentes caracterizaciones de sociedad serán las siguientes: Sociedad de la Información, emergida a principios del siglo XX, cuyo pilar es el procesamiento, distribución o utilización de la información, en la que distinguimos un primer momento, a principios del siglo XX que fue impulsado por los mass media (televisión, radio y prensa) y, un segundo, con la aparición de las TIC e Internet (a partir de 1980); Sociedad del Conocimiento, variante de la anterior, que aparecerá, junto con la anterior, cuando los integrantes de dicha sociedad acceden a la información y la conozcan; Sociedad Red (a partir de 1980), estructura social característica de la Sociedad de la Información; y Sociedad Digital o virtual (a partir del 2010), en la que los integrantes de la sociedad vivirán y desarrollarán su día a día en Internet, de forma virtual, y en la que la organización social (relaciones, trabajo, servicios, etc.) tendrá lugar en el espacio virtual. 


\section{LA SOCIEDAD DE CONTROL: EL PANÓPTICO DIGITAL, LA VIGILANCIA DIGITAL O VIRTUAL Y EL AUTO-CONTROL SOCIAL}

Como hemos mencionado, actualmente, la sociedad se caracteriza por ser digital, por vivir, cada vez más, en un espacio virtual. La sociedad, en gran medida, hace uso de aplicaciones o apps como Twitter, Facebook, WhatsApp, Instagram, Skype, LinkedIn y otras, servicios en streaming como Netflix o Spotify; usamos smart devices o dispositivos inteligentes, smartphones, pagos vía on-line, etc. Todo esto ha traído y está trayendo un cambio en el volumen de datos y fenómenos como el Big Data, cuya mayor característica es precisamente el volumen de los datos: las estimaciones de aumento de datos generados indican un crecimiento sin precedentes, debido a las redes sociales y a la movilidad que facilitan las redes inalámbricas y la telefonía móvil. Este incremento de datos determina un cambio de escala, pasando de terabytes a petabytes y zetabytes (Pérez Huerta, Edo Solana y Poveda Andrés, 2018). Todo ello supone que toda nuestra información esté en la red. Contribuimos a la Sociedad de la Información, no solo somos meros integrantes. Ello, además, tiene otra deriva: las empresas sacan beneficio económico de nuestros datos y venden información relativa a nuestras vidas, la información que dejamos en la red puede ser usada para localizarnos geográficamente, etc.

Tal como apunta Sancho López (2019: 309), las nuevas tecnologías inteligentes funcionan a partir de datos y metadatos que dan información sobre el qué, quién, cuándo, cómo, dónde, etc., permitiendo crear catálogos de ficheros de datos con el objetivo de explotarlos posteriormente con diversos fines. Estos datos pueden ser conseguidos de muchas maneras diferentes, por ejemplo, a través de las aplicaciones que descargamos en cualquier dispositivo (Harcourt, 2014).

Actualmente, todos nuestros datos quedan en la red, al desarrollar nuestra vida de manera digital. Todo ello debemos unirlo a lo ya comentado: la Sociedad de la Información se basa en el procesamiento, distribución o utilización de la información (Castells, 1997: 43) y la información estratégica es fuente del nuevo poder (Keohane, Nye, 1998); por lo tanto, somos nosotros quienes en nuestra interacción con la red dejamos nuestra información al alcance de todos; información que puede ser utilizada por cualquiera con fines de control, comerciales, económicos, etc. Es ahí donde confluirán la Sociedad de la Información, los datos a gran escala (Big Data) y la Sociedad Digital.

Como apunta Byung-Chul (2014a), la gran nube de información que cruza todos los datos de las redes sociales, llamada Big Data, brinda psicoperfiles de los usuarios que son usados para controlarlos. Uno de esos casos, por ejemplo, fue el "caso Cambridge Analytica (CA)» que hacía uso de datos de redes sociales con objetivos electorales, que podrían llevar a cambios en el poder y en la sociedad. Un caso parecido puede ser el de la geolocalización o localización geográfica de los integrantes de la sociedad mediante sus dispositivos digita- 
les. Teniendo en cuenta todo lo mencionado hasta aquí, podemos concluir que, a mayor información nuestra, mayor control.

Por todo ello, se podría decir que hoy en día estamos avanzando hacia una Sociedad de Control o Sociedad de la Transparencia (Deleuze, 1992; Byung-Chul, 2014a; Bauman y Lyon, 2013; Alcántara, 2008), que estamos impulsando nosotros mismos. Nuestro punto de partida será la vigilancia derivada de la digitalización.

Tal como afirman Bauman y Lyon (2013), se han desarrollado actualmente dos métodos paralelos de control y vigilancia, contrapuestos al tiempo que complementarios: la captación de información personal por parte de las redes sociales, y el empleo de drones para observar inadvertidamente todo lo que ocurre sobre la faz de la tierra. Toda esta información puede ser utilizada para un mayor control de cada integrante de la sociedad y, además, según el sociólogo, el desarrollo tecnológico contribuye a la ubicuidad de las prácticas de vigilancia en nuestras sociedades. Aparecerá, así, la «vigilancia líquida».

La nueva vigilancia o vigilancia líquida se basa en el procesamiento de la información, más que en lo que afirmaba Foucault (2009), y permite una nueva transparencia en la que no solamente los ciudadanos como tal sino todos nosotros, en cada uno de los roles que asumimos en nuestra vida cotidiana, somos constantemente controlados, observados, examinados, evaluados, valorados y juzgados (Bauman y Lyon, 2013: 10). Esta vigilancia, además, implica un control constante que excede los modelos cerrados de vigilancia; todo ello proporcionado por voluntad propia, con nuestros teléfonos móviles, GPS, tarjetas de crédito, redes sociales y demás (Santiere, 2018).

Dicho todo esto, nuestra primera conclusión será que la digitalización actual y el hecho de vivir digitalmente y en el espacio virtual lleva a la aparición de una vigilancia líquida: una vigilancia digital o vigilancia virtual que usará toda nuestra información para la vigilancia. De este modo, esta vigilancia líquida será omnipresente y etérea, ya que se hará de forma digital con el procesamiento de la información que los mismos integrantes de la sociedad ponen en manos de cualquiera, como consecuencia de la digitalización. Es cada vez más común lo que se ha venido a denominar la «vigilancia 2.0. A través de esta vigilancia se usan aplicaciones y herramientas digitales para acceder a la información en la red, mediante la búsqueda y filtrado de información que ofrece esta vigilancia virtual (vid. ADEGI, 2013)—. Así, ya no harán falta videocámaras o sistemas de videovigilancia, bastará con acceder a la red digital y a las tecnologías smart y hacer uso de toda la información que hemos dejado al alcance de cualquiera.

Aquí es donde aparecerá uno de los mayores problemas. Tal como expuso Bentham (2011) con el ejemplo de los guardias y los prisioneros, la posibilidad de ser permanentemente vigilados desarrolla auto-control; en la misma línea, la teoría del panóptico Foucault (2009): el surgimiento del auto-control de los sujetos debido a la presencia de vigilancia (la restricción física se sustituye por el auto-control). De este modo, la posibilidad de estar vigilado 
altera nuestro comportamiento. El hecho de estar vigilados supone la expectativa de un castigo si realizamos determinadas conductas, lo que trae consigo que se lleven a cabo las respuestas que provoquen consecuencias positivas o que eviten la imposición de un castigo o supongan consecuencias negativas. Todo ello nos puede llevar de ser una sociedad basada en la libertad, a una sociedad basada en el control (Remotti Carbonell, 2009), ya que una sociedad excesivamente vigilada jamás podrá ser libre (Alcántara, 2008).

Esta vigilancia, como hemos dicho, será líquida o digital, por lo que, partiendo de estas premisas sobre el control social y adaptándolas a la digitalización actual y el Big data, hablamos ya de un panóptico digital. En el panóptico digital el poder puede observar cada acción de todas las personas sin que sepan qué se está observando, cuándo o con qué propósito (Boyd, 2002: 64). La posibilidad de estar digitalmente vigilado desarrolla auto-control en los integrantes de la sociedad, por lo que la Sociedad Digital se acaba convirtiendo en una Sociedad de Control basada en el auto-control de los propios integrantes. Actualmente, somos nosotros los prisioneros y la digitalización es la torre de los guardias, en la que no llegamos a ver si los guardias están o no; así, aunque se produjera la vigilancia, podríamos no tener conocimiento de ello, porque la digitalización así lo permite (vigilancia digital, omnipresente y etérea); lo que vuelve a incidir en no saber si estamos siendo vigilados, llevándonos al auto-control.

El panóptico digital resulta una evolución del panóptico que deriva de la digitalización de la sociedad. Este concepto supera el "panóptico electrónico» que fue esbozado como el propio de sistemas de videovigilancia y cámaras en espacios públicos (Carracedo, 2002; y Ruiz Chasco, 2014). En esta idea, ya no hablamos de un control que tiene lugar en el espacio real con videocámaras en espacios públicos y demás, sino que se desarrolla en el espacio virtual, a través de nuestros datos en la red y nuestra huella digital.

En este panóptico digital (Galvis Cano, 2018), el control disciplinado se convertirá en control digital, la herramienta será la vigilancia líquida, y la disciplina estará en el ecosistema digital, en el ciberespacio, dentro de cada sociedad. Todos nuestros datos e información que dejamos en el espacio virtual (nuestra navegación, la huella digital, etc.) será utilizado con diversos fines (propios de la Sociedad de la Información) y, además, nos hará transparentes y el panóptico digital se convertirá en un elemento esencial de control (Castells, 2017).

Todo ello proviene de una concatenación de premisas analizadas hasta aquí: la sociedad actual vive de manera digital y, en consecuencia, toda su información queda en la red; dicha información y la digitalización derivan en una vigilancia líquida o digital, y, así, la posibilidad de vigilancia nos lleva a una sociedad controlada, al preguntarse la ciudadanía si están siendo o no vigilados por los guardias digitales o virtuales. Las aplicaciones que descarguemos en cualquier dispositivo, el Big Data, la geolocalización, nuestros hábitos de búsqueda en internet y demás suponen que la ciudadanía tenga la constante percepción de estar bajo una vigilancia omnipresente (vigilancia líquida), una vigilancia desde una torre de guardias 
digital que no sabemos si están o no, pero que deriva en que la ciudadanía limite sus comportamientos y se imponga a sí misma un auto-control en beneficio del poder.

Asimismo, en el panóptico digital, el auto-control no será por una vigilancia por parte del poder, sino que somos nosotros mismos quienes generamos esa posibilidad de estar vigilados a través de nuestra vida digital, que acarreará la posibilidad de ser vigilados mediante dichos recursos digitales y toda nuestra información en la red; supone, en sí, una especie auto-vigilancia que deriva en auto-control; la sociedad, al final, se acaba vigilando a sí misma (un auto-panóptico). Estamos, por tanto, ante una paradoja del panóptico: no hacen falta torres de guardias o videocámaras de vigilancia (por parte del poder) para que la ciudadanía tenga la sensación de vigilancia; el mero hecho de vivir en el universo digital o virtual (utilizar apps, redes sociales, etc.) nos hace al mismo tiempo temer que mediante esos datos estamos siendo vigilados (los integrantes de la sociedad construyen la idea de vigilancia y ellos mismos se auto-controlan).

Como afirma Byung-Chul (2013: 34), en el enjambre digital actual ya no somos meros receptores y consumidores pasivos, sino emisores y productores activos. En la Sociedad Digital actual, son los integrantes de la sociedad los que producen la información y los que impulsan el control social: suponemos el principal pilar de la Sociedad de la Información y de la Sociedad Digital, pero, también, de la Sociedad de Control. Además, cada integrante de la sociedad deja de tener su espacio íntimo o vida interior y pasamos a la desnudez de cada integrante; aquellos que se preocupan de su intimidad acaban excluidos o relegados (Enriquez, 2004: 49).

La ciudadanía es quien deja en manos de cualquiera su información personal, lo que supone la posibilidad de que cualquiera pueda acceder y controlar a dicha persona: es cada integrante de la sociedad quien construye su propia torre que le lleva al auto-control, y, además, con su consentimiento y aprobación. El poder consigue, de esta manera, un control social sin necesidad de realizar ningún tipo de acción: no hacen falta torres, guardias o videocámaras, únicamente el rastro digital que los mismos integrantes de la sociedad crean.

Aún más, en el panóptico digital, la posibilidad de vigilancia no vendrá únicamente por parte de los poderes: cualquiera podrá acceder y hacer uso o controlar a cualquier integrante de la sociedad, por lo que la posibilidad de vigilancia se incrementará (ya no solo la posibilidad de vigilancia pública, también privada).

Toda esta información almacenada en enormes bases de datos, junto a la digitalización y las nuevas tecnologías que, además, se lleva a cabo de forma masiva por parte de administraciones públicas y empresas privadas, nos lleva a lo que se ha denominado Dataveillance (vigilancia de datos): la normalización social de la cultura de la vigilancia (Sancho López, 2019: 309). Esto es precisamente lo que acaba siendo la vigilancia omnipresente digital o virtual, es decir, la vigilancia líquida de Bauman, pero con un añadido extra que nos 
trae la revolución tecnológica (y el hecho de vivir en el espacio virtual) y el valor de la información personal (característica de la actual Sociedad de la Información).

Más aún, el panóptico digital, al ser digital o virtual, trasciende de los límites espacio-temporales. Así, al nuevo panóptico se le ha denominado el panspectrum, término acuñado por Braman (2006), que supone una evolución del panóptico: este concepto no se limita a una vigilancia en la que vigilante y vigilado coincidan en tiempo y espacio, sino que se da aunque ambos estén a larga distancia o en tiempo distinto (búsquedas en internet, cookies, el timeline, movimientos bancarios...). Por tanto, tenemos una probable vigilancia líquida, Dataveillance (vigilancia de datos), que nos supone un panspectrum; una vigilancia que nosotros creamos y permitimos, con un auto-control que nosotros generamos y en el que caemos.

De este modo, tal como apunta Boyd (2002: 64), podrá darse un panóptico digital ideal donde los usuarios de Internet se sentirán observados por una figura externa de autoridad e, incluso, algunas veces se comunica al usuario que está siendo observados, quedando en evidencia la cantidad de datos almacenados sobre este (Boyd, 2002: 52); ello deriva en que el propio usuario se impondrá límites a sí mismo por un sentimiento de supervisión de un ente superior (Díaz-Bizkarguenaga, 2018: 59). Resultado: cada uno es panóptico de sí mismo (Byung-Chul, 2014b: 35).

Además, el panóptico digital creado por los integrantes de la sociedad, cuenta con la aprobación social de estos (la auto-vigilancia que hemos mencionado): los integrantes saben que con los datos masivos de los dispositivos y nuevas tecnologías se puede mantener un control y una vigilancia, pero, aun sabiéndolo, siguen dejando sus información personal y siguen manteniendo el mismo comportamiento; con la consciencia (o no) de que son ellos mismos los que crean el panóptico digital y los que pueden acabar con él.

En relación al poder, el control social derivado del panóptico digital, puede venir, entre otros, del uso de toda la información junto a la tecnología y transformación digital en el ámbito de la seguridad (Froment, 2002). Toda la información (bases de datos, imágenes, localización, comunicación, etc.) puede ser utilizada para controlar a la sociedad, especialmente en nombre de la seguridad pública: un sistema que usa datos creados por otros para controlar las personas, los movimientos y sus actividades (Nunn, 2001). Las nuevas tecnologías digitales favorecen el control de la autoridad y el monitoreo de las actitudes ciudadanas (Morozov, 2013).

Así, la ciudadanía se aplicará el auto-control al saber que sus datos pueden estar siendo usados (o no) para controlar si el ciudadano ha cumplido (o no); es decir, no veremos ni sabremos si se está haciendo un uso constante de vigilancia digital (la torre), pero, por si acaso, adaptaremos nuestro comportamiento por dicha posibilidad (auto-control derivado de una vigilancia digital). La sensación de vigilancia que deriva de la digitalización acarrea el control de la ciudadanía. 
Castells (2009) sigue una tesis parecida al afirmar que la digitalización total de la comunicación permite la creación de una vasta red global de vigilancia y de control, la mayor de la historia. Según él, los Estados lo que quieren es vigilancia, control y poder, y vivimos en una sociedad con la información como eje, lo cual facilita dicho control, totalmente digitalizada. Apunta el sociólogo que la digitalización será pieza clave en dicho control, ya que el proceso de formación y ejercicio de las relaciones de poder se transforma radicalmente en el contexto del auge de las redes digitales de comunicación globales (Castells, 2009: 24 y 25).

Otra de las consecuencias del panóptico digital en relación al poder, será la paradoja de la transparencia y la falsa idea de control del poder (Byung-Chul, 2014a): una sociedad que intenta ser transparente con objeto de reducir el poder y controlarlo, y que acaban siendo controlados los ciudadanos. Así, ningún otro lema domina hoy tanto el discurso público como la transparencia (Byung-Chul, 2014a: 11); un discurso anclado en las libertades para acceder a y difundir información, reducir la corrupción y controlar el poder político. Todo ello se basa en la información que es accesible, pero toda esa información es producida por todos los integrantes de la sociedad y contiene, por lo tanto, información de todo tipo y de todo integrante. De este modo, toda esa información hace que estemos en una sociedad en la que somos vigilados no desde un centro único de mirada despótica, sino desde una vigilancia que tiene su origen en todas partes (vigilancia líquida de Bauman, una vigilancia digital); así, la Sociedad de la Transparencia se convierte en una Sociedad del Control, en la que sus propios integrantes colaboran de forma activa en su construcción y conservación, siendo sujetos de control y productores del mismo. Y concluimos: esta vigilancia total degrada la Sociedad de la Transparencia hasta convertirla en una inhumana Sociedad del Control (Byung-Chul, 2014a: 90). Supone, en suma, una paradoja: la idea de controlar el poder mediante la transparencia, acaba derivando en un control social, u auto-control de la ciudadanía que sigue lo que dicta el poder, al no saber si está siendo vigilada o no.

La Sociedad de la Transparencia o sociedad de la exposición hacia la que la sociedad ha avanzado, además, deriva de un fetichismo digital impuesto por las redes sociales y, sobretodo, porque representa la única forma de existir. De este modo, todo se mide en su valor de exposición (Byung-Chul, 2014a: 29).

Por otro lado, Morozov (2013) apunta a efectos positivos y a la generalización de un panóptico invertido: si los integrantes de la sociedad se sienten constantemente observados nos sentiríamos compelidos a ser nuestra mejor versión, estando en un mundo donde las malas decisiones ya no son una opción, no podemos decidir sino ser buenos (Morozov, 2013: 290); de este modo, el imperativo categórico kantiano pasa de ser una elección voluntaria de cada integrante a ser un resultado de la observación ajena. Esta tesis es aceptable partiendo de la vigilancia: los integrantes se sienten obligados a dar su mejor versión a consecuencia de una vigilancia (se auto-controlan), vigilancia que ellos mismos impulsan, pero, al fin y al cabo, el hecho de que el imperativo categórico sea generado y no elegido, hace que estemos ante integrantes auto-controlados y no autónomos, cuya capacidad de elección o libre albedrío acaba siendo laminada por la posibilidad de ser vigilados. Esto deriva 
en que ya no existe un mero auto-control (no hacer conductas que tendrán respuesta negativa, pero seguir siendo libre a la hora de elegir las conductas restantes), sino que habrá unas conductas imperativas de obligado cumplimiento social.

La Sociedad de Control resulta una degradación de la Sociedad Digital y se caracterizará por lo siguiente: todos los datos y la información de cada integrante de la sociedad en su interacción en el espacio virtual traerán la posibilidad de una vigilancia virtual, etérea y omnipresente (fomentada por los mismos integrantes), que llevará al auto-control y, por ende, a constituirse en una sociedad controlada; un control que será validad y aprobado por la misma sociedad que es quien creará toda la estructura de vigilancia que estará a disposición de cualquiera.

Además, en la Sociedad de Control, no solo habrá la mencionada posibilidad de vigilancia, sino, también, la vigilancia real que se puede llevar a cabo con el uso de los instrumentos digitales: la vigilancia digital (digital surveillance), que supone el uso de la tecnología digital para controlar a otras personas, ciudadanos y la vida de un Estado, en general (Korstanje, 2018; Korstanje y Skoll, 2018). Por lo que en la Sociedad de Control la fusión entre la información (Sociedad de la Información) y el espacio virtual (Sociedad Digital) tendrá dos consecuencias: una ciudadanía auto-controlada (panóptico digital) y una ciudadanía realmente controlada (vigilancia real por medios digitales).

Asimismo, se exteriorizan dos nuevas amenazas La primera: la norma general de vida privada supone mantener en privado los aspectos íntimos y la de la vida pública supone la transparencia y la publicidad, pero, en este contexto social, la vida privada acaba siendo pública y la vida pública acaba siendo privada (paradoja de la transparencia). Los integrantes quedan públicamente expuestos (además de controlados) y la vida pública deja de ser transparente a la ciudadanía; el poder que debe ser controlado acaba sin control y los que deben ser controladores acaban siendo controlados. Y, la segunda, sería las mencionadas conductas imperativas de obligado cumplimiento social, derivadas de la posibilidad de vigilancia, que supera el mero auto-control.

Por último, en palabras de Sancho López (2019: 313), supondrán un riesgo, asimismo, las posibles prácticas discriminatorias que puedan derivarse de la ideología del algoritmo que orienta el Big Data: la posible exclusión de colectivos e individuos, así como las limitaciones en el disfrute de derechos y libertades públicas, pueden derivar en un proceso de segmentación social. De este modo, tal como defienden algunos autores, aparecerán un nuevo fenómeno: la sociedad de clases digital (Byung-Chul, 2014b: 99).

\section{CONCLUSIONES}

Con todo lo analizado debemos hacer unas últimas reflexiones. Todas ellas tendrán el mismo pilar básico: la información, los datos. 
Hemos partido de un tipo de sociedad (Sociedad de la Información) que tiene como pilar el proceso de captar, procesar y comunicar la información: el procesamiento, almacenamiento, distribución y utilización de datos. Este modelo de sociedad está hoy más vivo que nunca por las nuevas tecnologías y la revolución digital que han traído un aumento en el volumen, cantidad, calidad y rapidez de datos, revitalizando la información y convirtiéndola, aún más, en el pilar de la sociedad (la información es el petróleo del siglo xxi), con lo que la Sociedad de la Información está constantemente llegando a su apogeo.

En este contexto, la información se caracteriza por poseer un valor especialmente alto desde el punto de vista comercial, electoral, de control, etc. A ello le añadimos la digitalización y su transformación: la sociedad ha pasado de una mera interconexión entre dispositivos a vivir digitalmente, una sociedad construida sobre un entorno muy diferente al mundo físico (espacio virtual), donde los servicios se prestan de forma digital o virtual y donde los integrantes de la sociedad realizan sus actividades cotidianas también de forma digital, lo que define la Sociedad Digital. El hecho de realizar todas las facetas de la vida cotidiana en la red provoca que dejemos nuestra información en esta; de este modo, los integrantes de la sociedad se convierten en productores de información.

Todo ello tendrá una consecuencia: la vigilancia digital o vigilancia líquida; una vigilancia virtual, omnipresente y etérea que no se basará en guardias o videocámaras, sino en la información que los mismos integrantes de la sociedad han producido. Ello nos derivará en un panóptico digital, en el que la posibilidad de dicha vigilancia traerá consigo un autocontrol social, pero, además, al ser una vigilancia omnipresente o líquida estaremos en un constante auto-control. En dicho panóptico digital, asimismo, ya no hará falta una posible vigilancia del poder, sino que la ciudadanía, como productores de información, contribuye a la propia vigilancia, con lo que acaba, paradógicamente, auto-vigilada y auto-controlada (auto-panóptico). Dicho auto-control, igualmente, ya no será un mero auto-control donde la ciudadanía mantenga una capacidad de elección, sino que supondrá el cumplimiento de unas conductas estándares que derivará de la vigilancia que los mismos ciudadanos han impulsado.

A ello se le une la vigilancia real que se efectúa por medio del espacio virtual y la información de los integrantes de la sociedad, la dataveillance: la normalización social de la cultura de la vigilancia. Así, la Sociedad de Control se caracterizará por la desnudez de los integrantes de la sociedad, cuya exposición en la red traerá consigo una vigilancia real, por un lado, y una posibilidad de vigilancia creada por ellos mismos que derivará en auto-control; una ciudadanía cada vez más controlada.

Por último, actualmente, la transparencia se degradará hasta el punto de que lo público dejará de ser transparente y lo privado comenzará a ser público. Habrá, así, un poder sin control, con unos ciudadanos controlados. 


\section{REFERENCIAS BIBLIOGRÁFICAS}

ADEGI (2013). Vigilancia 2.0 (gestión eficaz de la información). Accesible en: https://www. adegi.es/adegi/vigilancia-20-gestion-eficaz-informacion/

Alcántara, J. F. (2008). La sociedad de control. Barcelona: El Cobre Ediciones. Accesible en: https://www.versvs.net/la-sociedad-de-control/

Alfonso Sánchez, I. R. (2016). La Sociedad de la Información, Sociedad del Conocimiento y Sociedad del Aprendizaje. Referentes en torno a su formación. Bibliotecas. Anales de Investigación, vol. 12, n. ${ }^{\circ}$ 2, pp. 235-243.

Aristovnik, A. (2014). Development of the information society and its impact on the education sector in the EU: efficiency at the regional (NUTS 2) level. Turkish online journal of educational technology, vol. 13. n. ${ }^{\circ}$ 2. pp. 54-60.

Barney, D. (2003). The Network Society. Cambridge: Polity.

Bauman, Z., y Lyon, D. (2013). Vigilancia líquida. Barcelona: Paidós.

Bell, D. (1976). El advenimiento de la sociedad postindustrial. Madrid: Alianza.

Bentham, J. (2011). Panóptico. Madrid: Círculo de Bellas Artes.

Blázquez Entonado, F. (2001). Sociedad de la Información y Educación. Mérida: Junta de Extremadura.

Boyd, D. M., y Ellison, N. B. (2007). Social Network Sites. Definition, history, and Scholarship. Journal of Computer-Mediated Communication, vol. 13, n. ${ }^{\circ} 1$.

Boyd, D. (2002). Faceted Id/entity: Managing Representation in a Digital World. MIT Master's Thesis, Cambridge. Accesible en: https://smg.media.mit.edu/people/danah/ thesis/danahThesis.pdf

Braman, S. (2006). Change of state: Information, policy, and power. Cambridge (Massachusetts): MIT Press.

Brito-Alvarado, L. X., y Capito Álvarez, S. P. (2016). Subjetividades on line: Entre la vigilancia y la autorreferencia global. Sociología y tecnociencia: Revista digital de sociología del sistema tecnocientífico, vol. 2, n. ${ }^{\circ}$ 6, 2016, pp. 17-29. Accesible en: https://revistas. uva.es/index.php/sociotecno/article/view/657

BSA (The Software Alliance) (2015). Informe ¿Por qué son tan importantes los datos? Accesible en: https://data.bsa.org/wp-content/uploads/2015/10/BSADataStudy_es.pdf 
Byung-Chul, H. (2013). En el enjambre. Barcelona: Heder.

Byung-Chul, H. (2014a). La sociedad de la transparencia. Barcelona: Heder.

Byung-Chul, H. (2014b). Psicopolítica. Barcelona: Heder.

Carracedo, J. D. (2002). La Vigilancia en las sociedades de la información. ¿Un panóptico electrónico? Política y Sociedad, vol. 39, n. ${ }^{\circ}$ 2, pp. 437-455.

Casas Pérez, M. L. (2010). México: sociedad de la información o sociedad del conocimiento. Virtualis: revista de cultura digital, vol. 1, n. $^{\circ}$ 1, (Ejemplar dedicado a: Hacia la Sociedad de la Información), pp. 22-43.

Castelfranchi, C. (2007). Six critical remarks on science and the construction of the knowledge society. Journal of Science Communication, n. ${ }^{\circ}$ 6. Accesible en: https://doi. org/10.22323/2.06040303

Castells, M. (1997). La era de la información: Economía, sociedad y cultura. Vol. 1. La sociedad red (versión castellana de Carmen Martínez Gimeno). Madrid: Alianza.

Castells, M. (2001). Internet y la Sociedad Red. La Factoria, la revista social. Accesible en: https://revistalafactoria.org/articulos/2018/6/4/internet-y-la-sociedad-red

Castells, M. (2002a). La dimensión cultural de Internet. Universitat Oberta de Catalunya. Accesible en: https://www.uoc.edu/culturaxxi/esp/articles/castells0502/castells0502. html

Castells, M. (2002b). La Galàxia Internet. Barcelona: Rosa dels Vents.

Castells, M. (2006). La sociedad red: una visión global. Madrid: Alianza.

Castells, M. (2007). Innovación, libertad y poder en la era de la información. En De Moraes, D. (coord.), Sociedad Mediatizada. Barcelona: Gedisa.

Castells, M. (2009). Comunicación y poder. Madrid: Alianza.

Castells, M. (2014). El impacto de internet en la sociedad: una perspectiva global. En VV.AA., C@mbio.19 ensayos fundamentales sobre cómo internet está cambiando nuestras vidas. Madrid: Turner. Accesible en: https://www.bbvaopenmind.com/wpcontent/uploads/2014/03/BBVA-Comunicaci\%C3\%B3n-Cultura-Manuel-Castells-Elimpacto-de-internet-en-la-sociedad-una-perspectiva-global.pdf

Castells, M. (2017). El panóptico digital. Vanguardia dossier, n. ${ }^{\circ}$ 63, pp. 74-77. 
Castells, M. (2020). La nueva sociedad red. La Vanguardia. Accesible en: https://www. lavanguardia.com/opinion/20200118/472937590818/la-nueva-sociedad-red.html

Castells, M., y Cardoso, G. (1996). The network society. Washington: Center for Transatlantic Relations, Paul H. Nitze School of Advanced International Studies, Johns Hopkins University.

CCOO (2017). La Digitalización y la Industria 4.0. Impacto industrial y laboral. Madrid.

Cervantes Benavides, L. E. (2007). La sociedad del conocimiento y los servicios de educación superior en la globalización. Universidades, n. 34 , pp. 25-40.

Contreras Gómez, C. (2017). El papel del Gobierno en la era digital. Un enfoque de economía pública. Madrid: Editorial Universitaria Ramón Areces.

Crawford, S. (1983). The Origin and Development of a Concept: The Information Society. Bull Med Libr Assoc., 71(4), pp. 380-385.

Deleuze, G. (1992). Postscript on the Societies of Control. October, vol. 59, pp. 3-7.

Díaz-Bizkarguenaga, K. (2018). La construcción de la identidad vasca bajo el panóptico digital: retos para una democracia digital. En Candón Mena, J. (ed.), Actas del II Congreso Internacional Move.net sobre Movimientos Sociales y TIC. Sevilla: Universidad de Sevilla, Grupo Interdisciplinario de Estudios en Comunicación, Política y Cambio Social (COMPOLÍTICAS).

Drucker, P. F. (1969). The Age of Discontinuity: Guidelines in Our Changing Society. New York: Harper and Row.

Drucker, P. F. (1993). The Rise of the Knowledge Society. Wilson Quaterly, vol. 17, n. ${ }^{\circ} 2$.

Echevarría, J. (1999). Los señores del aire. Telépolis y el tercer entorno. Barcelona: Desrino.

Echevarría, J. (2000). Conocimiento en el medio ambiente digital. Nueva Revista, n. ${ }^{0}$ 70, pp. 25-29. Accesible en: https://www.nuevarevista.net/destacados/conocimiento-enel-medio-ambiente-digital/

Enriquez, E. (2004). L'idéal type de l'individu hypermoderne: l'individu pervers?. En Aubert, N. (ed.), L'Individu hypermoderne. Toulouse: Érès.

Foucault, M. (2009). Vigilar y castigar. Nacimiento de la prisión. Madrid: Siglo XXI.

Froment, J.-C. (2002). Les noves tecnologies de seguretat i la «societat de control». Revista Catalana de Seguretat Pública, n. ${ }^{\circ}$ 10, pp. 23-37. 
Galvis Cano, L. (2018). El Panóptico digital de la protección de datos personales en Colombia. Revista Temas: Departamento de Humanidades Universidad Santo Tomás Bucaramanga, n. ${ }^{\circ}$ 12, pp. 125-140.

García Manjón, J. V. (2010). Innovar en la era del conocimiento. Claves para construir una organización innovadora. Oleiros (A Coruña): Netbiblio.

Hamelink, C. (2003). Human Rights for the Information Society. En Girard, B., y Ó Siochru, S. (eds.), Communicating in the Information Society. Ginebra: United Nations Research Institute for Social Development. Accesible en: http://www. unrisd.org/80256B3C005BCCF9/(httpAuxPages)/5DCA28E932BB8CFDC1256E2400 29A075/\$file/InfoSoc.pdf

Harcourt, B. (2014). Governing, Exchanging, Securing: Big Data and the production of a digital knowledge. Columbia Public Law Research Paper No. 14-390 (2014), Public Law and Legal Theory Working Paper Group, Columbia Law School. Accesible en: https:// scholarship.law.columbia.edu/faculty_scholarship/1860

Harrison, S. R., y Dourish, P. (1996). Re-Place-ing Space: the roles of place and space in collaborative systems. Proceedings of the 1996, ACM conference on Computer supported cooperative work (Conference Paper), Boston (Massachussets), pp. 67-76. Accesible en: https://dl.acm.org/doi/10.1145/240080.240193

IIS (2001). Conversación con Manuel Castells. Berkeley (California): Institute of International Studies (IIS), University of California. Accesible en: http://globetrotter.berkeley. edu/people/Castells/castells-con4.html

Keohane, R. O., y Nye, J. S. (1998). Power and Interdependence in the Information Age. Foreign Affairs, vol. 77, n. ${ }^{\circ} 5$.

Korstanje, M. E. (2018). A Paradoxical World and the Role of Technology in Thana-Capitalism. En Khosrow-Pour, M. (ed.), Encyclopedia of Information Science and Technology (pp. 4761-4773). Hershey (Pensilvania, EEUU): IGI Global.

Korstanje, M. E., y Skoll, G. (2018). Technology and Terror. En Khosrow-Pour, M. (ed.), Encyclopedia of Information Science and Technology (pp. 3637-3653). Hershey (Pensilvania, EEUU): IGI Global.

Kuwahara, T., Kamishima, J., y Komatsu, S. (1964). Joho shakai no soshiorogii (Sociology in information societies). Hoso Asahi, pp. 19-39.

Lafuente, M., y Genatios, C. (2006). ¿Sociedad del Conocimiento o Sociedad de la Información?. Comunicación: estudios venezolanos de comunicación, n. 133, 2006, pp. 20-24. 
Llano, A. (2000). La hora de la Sociedad de la Inteligencia. Nueva Revista, n. ${ }^{\circ}$ 70, pp. 162169. Accesible en: https://www.nuevarevista.net/destacados/la-hora-de-la-sociedadde-la-inteligencia/

Machlup, F. (1962). The Production and Distribution of Knowledge in the United States. Princeton (Nueva Jersey): Princeton University Press.

Marrero Rodríguez, G., y Santiago García, O. (1999). Aprender en la sociedad del conocimiento. Anuario de Filosofía, Psicología y Sociología, n. ${ }^{\circ}$ 2, pp. 97-109.

Martire, Antonio (2013). Espacio físico y espacio virtual en prácticas educativas. Trabajo Final de Máster, Universidad Autónoma de Barcelona (UAB). Accesible en: https:// ddd.uab.cat/pub/trerecpro/2013/hdl_2072_216837/TFM-A.Martire-correcto.pdf

Masuda, Y. (1981). The Information Society: As Post-industrial Society. Tokyo: IIS.

Masuda, Y. (1984). La sociedad informatizada como sociedad post-industrial. España: Fundesco, Tecnos.

Morozov, E. (2013). To Save Everything, Click Here. Technology, solutionism and the urge to fix problems that don't exist. Londres: Allen Lane.

Nunn, S. (2001). Police technology in cities: changes and challenges. Technology in Society, vol. 23, n. ${ }^{0}$ 1, pp. 11-27. Accesible en: https://doi.org/10.1016/S0160-791X(00)00033-6

Papacharissi, Z. (2010). The Networked Self: Identity, Community, and Culture on Social Networking Sites. Londres: Routledge.

Penty, A. J. (2004). Post Industrialism. Montana: Kessinger Publishing.

Pérez Huerta, P., Edo Solana, M. D., y Poveda Andrés, J. L. (2018). Cuadernos de Gestión Sanitaria. Número 4. Datos de Vida Real y Macrodatos: Su incorporación a la evaluación. Madrid: Fundación Gaspar Casal. Accesible en: http://fundaciongasparcasal. org/aeets/publicaciones/cuadernos-gestion-sanitaria-numero-4-datos-de-vida-real-ymacrodatos.pdf

Pineda de Alcázar, M. (2018). La Internet de las Cosas, el Big Data y los nuevos problemas de la comunicación en el siglo xxi. Mediaciones Sociales, vol. 17, pp. 11-24.

Porat, M. (1977). The Information Economy: Definition and Measurement. Washington DC: U.S. Government Printing Office (Office of Telecommunications, U.S. Department of Commerce; OT special publication 77-12). 
Remotti Carbonell, J. C. (2009). De la sociedad de la libertad a la sociedad del control. En Pérez Francesch, J. L. (ed.), Libertad, seguridad y transformaciones del Estado. Barcelona: Institut de Ciències Polítiques i Socials.

Rooney, J. (2014). Proceedings of the 11th International Conference on Intellectual Capital, Knowledge Management and Organizational Learning. UK: Academic Conferences and Publishing International Limited.

Ruiz Chasco, S. (2014). Videovigilancia en el centro de Madrid: ¿Hacia el panóptico electrónico? Revista Teknokultura, vol. 11, n. ${ }^{\circ}$ 2, pp. 301-327.

Santiere, J. (2018). El panóptico de Foucault y el post-panóptico de Bauman. El Sigma. com. Accesible en: https://www.elsigma.com/subjetividad-y-medios/el-panoptico-defoucault-y-el-post-panoptico-de-bauman/13500

Salvat Martinrey, G., y Serrano Martin, V. (2011). La Revolución Digital y la Sociedad de la Información. Zamora: Comunicación Social.

Sancho López, M. (2019). Internet, Big data y nuevas tecnologías: repercusiones y respuestas del ordenamiento jurídico. Cuadernos electrónicos de filosofía del derecho, n. ${ }^{\circ} 39$, pp. 307-321.

Sanz-Magallón, J. M. (2000). ¿Qué es la sociedad del conocimiento?. Nueva Revista, n. ${ }^{\circ}$ 70, pp. 9-15. Accesible en: https://www.nuevarevista.net/destacados/que-es-la-sociedaddel-conocimiento/

Sen, A. (2000). Development as Freedom. New York: Anchor Books.

Steinmueller, E. W. (2002). Las economías basadas en el conocimiento y las tecnologías de la información y la comunicación. Revista Internacional de Ciencias Sociales. Organización de Estados Americanos, n. ${ }^{0}$ 171. Accesible en: http://www.oei.es/salactsi/ steinmuller.pdf

Toniatti, R. (1991). Libertad Informática y Derecho a la Protección de Datos Personales: Principios de Legislación Comparada. Revista Vasca de Administración Pública, n. ${ }^{\circ} 29$, pp. $139-164$.

UNESCO (2003). Towards Knowledge Societies. An Interview with Abdul Waheed Khan. World of Science, vol. 1, n. ${ }^{\circ}$ 4. Accesible en: http://www.unesco.org/new/en/ communication-and-information/resources/news-and-in-focus-articles/all-news/ news/towards_knowledge_societies_an_interview_with_abdul_waheed/ 
Sociedad de la Información, Sociedad Digital, Sociedad de Control | Andoni Polo Roca

Uriarte, L. M., y Acevedo, M. (2018). Sociedad Red y Transformación Digital: hacia una evolución de la consciencia de las organizaciones. Economía industrial, Ejemplar dedicado a: Nuevos Paradigmas Organizativos (I), pp. 35-49.

Van Dijk, J. (2006). The network society: Social aspects of new media. Londres: Sage Publications Ltd.

Vázquez Vela, F. J. (2011). La revolución digital y la sociedad de la información. Questiones publicitarias: revista internacional de comunicación y publicidad, n. ${ }^{\circ}$ 16, pp. 140-143.

Webster, F. (1994). What information society?. Information society, vol. 10, pp. 1-23. 